\title{
Beyond Framingham and coronary calcium: Predicted lifetime risk identifies unfavorable risk trajectories
}

\author{
Andre R. M. Paixao, MD, ${ }^{a}$ and Jarett D. Berry, $M D, M S^{a, b}$ \\ a Division of Cardiology, Department of Internal Medicine, University of Texas Southwestern \\ Medical Center, Dallas, TX \\ ${ }^{\mathrm{b}}$ Department of Clinical Sciences, University of Texas Southwestern Medical Center, Dallas, TX
}

Received Dec 4, 2013; accepted Dec 4, 2013

doi: 10.1007/s12350-013-9834-8

Risk estimation represents the cornerstone of prevention practice, reflecting the consistent observation that the benefit of treatment is proportional to the baseline absolute risk. ${ }^{1-3}$ Although this principle can be applied to any clinical scenario, it is particularly relevant for the prevention of atherosclerotic cardiovascular disease (ASCVD) with statins. Clinical trial data have consistently shown that statins are associated with a consistent $30 \%-50 \%$ relative risk reduction in both primary and secondary prevention populations depending on the dose and type of statin studied. However, the absolute risk reduction varies dramatically, reflecting the overall absolute risk level of the population studied.

Therefore, because of the central importance of absolute risk for ASCVD, there has been substantial interest in the improvement of risk estimation strategies in recent years. In general, traditional risk factors such as age, gender, cholesterol, blood pressure, diabetes, and smoking status represent the most important determinants of ASCVD risk. The 2013 cardiovascular risk assessment guidelines released by the American College of Cardiology and the American Heart Association continue this same paradigm and recommend moderate

Funding Dr Berry receives funding from the Dedman Family Scholar in Clinical Care endowment at University of Texas-Southwestern Medical Center; Grant K23 HL092229 from the National Heart, Lung, and Blood Institute; and Grant 13GRNT14560079 from the American Heart Association. Dr Berry is a member of the Speaker's Bureau for Merck \& Co. The other authors report no conflicts and no associations with industry.

Reprint requests: Jarett D. Berry MD, MS, Division of Cardiology, Department of Internal Medicine, University of Texas Southwestern Medical Center, 5323 Harry Hines Blvd, Dallas, TX 75390-8830; jarett.berry@utsouthwestern.edu

J Nucl Cardiol 2014;21:42-5.

$1071-3581 / \$ 34.00$

Copyright ( 2013 American Society of Nuclear Cardiology. to high dose statin therapy for individuals with prior cardiovascular disease (CVD), diabetes, low density lipoprotein cholesterol $>190 \mathrm{mg} \cdot \mathrm{dL}^{-1}$ or predicted 10 -year risk $\geq 7.5 \%$. ${ }^{4,5}$

However, it is now well accepted that coronary artery calcium (CAC) represents a significant and robust determinant of short-term cardiovascular risk. CAC is an indirect measurement of the overall burden of coronary atherosclerosis and improves short-term risk prediction to a greater extent than most other biomarkers. ${ }^{6,7}$ The ability of CAC to improve short-term risk prediction is now supported by robust evidence and current guidelines support the use of CAC screening as a guide to statin treatment decisions.

Although both traditional risk factors and CAC represent effective strategies for short-term absolute risk estimation that can serve as a guide to statin treatment decisions, they by definition do not consider long-term or lifetime risk for CVD. This represents an important weakness because a wealth of evidence suggests that atherosclerosis is not a short-term process but rather reflects the cumulative exposure to risk factors across the lifespan. For years, it has been well established that atherosclerosis begins in adolescence and results from early life exposure to traditional risk factors. ${ }^{8-10}$ This process persists across the lifespan and into older age such that the cumulative risk factor burden present in early and middle-age translates into a lifelong accumulation of atherosclerosis.

In the Cardiovascular Lifetime Risk Pooling Project, we included more than 250,000 participants from 18 cohorts in order to create robust estimates of lifetime risks for ASCVD across age, sex, race, and risk factor strata. ${ }^{11}$ For example, among participants who were 45 years of age at risk factor ascertainment, those with an all optimal risk factor profile (total cholesterol level $<180 \mathrm{mg}$ per deciliter; blood pressure $<120 \mathrm{~mm} \mathrm{Hg}$ 
systolic and $80 \mathrm{~mm} \mathrm{Hg}$ diastolic; nonsmoking status; and non-diabetic status) had substantially lower risks of total ASCVD than participants with two or more major risk factors (see Figure 1). These associations between middle-age risk factor strata and lifetime ASCVD risk were consistent across birth cohorts, suggesting that the presence or absence of traditional risk factor strata in middle-age represents the most important determinant of ASCVD risk across the lifespan.

Stated another way, in spite of its clinical utility, short-term absolute risk estimation strategies that incorporate traditional risk factors, CAC, or other biomarkers are fundamentally at odds with the biology of the atherosclerotic process. Thus, lifetime risk estimation represents an important supplement to short-term risk estimates that can provide insight into an important aspect of ASCVD risk.

Because short-term and long-term risk estimation strategies each provide a unique perspective, there has been much interest in their complementary nature and the apparent conflict between them. In particular, the vast majority of adults less than 50 years of age are at "low risk" in the short-term, regardless of their risk factor burden. This reflects the dominant role of age in short-term risk equations. However, because of the strong associations between middle-age risk factor

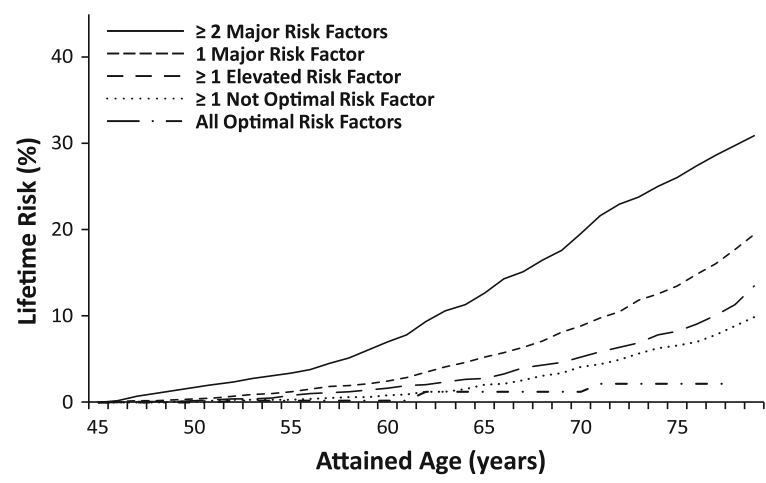

Figure 1. Lifetime risk for cardiovascular disease mortality among men age 45 years in the Cardiovascular Lifetime Risk Pooling Project $(\mathrm{N}=17,315)$. Figure modified from Berry et al. " "Optimal Risk Factor" was defined as total cholesterol $<180 \mathrm{mg} \cdot \mathrm{dL}^{-1}$, untreated blood pressure $<120 /<80 \mathrm{~mm} \mathrm{Hg}$, non-smoker, and non-diabetic. "Not Optimal Risk Factor" was defined as non-smoker and non-diabetic with total cholesterol $180-200 \mathrm{mg} \cdot \mathrm{dL}^{-1}$, or untreated systolic blood pressure $120-140 \mathrm{~mm} \mathrm{Hg}$ or untreated diastolic blood pressure $80-90 \mathrm{~mm} \mathrm{Hg}$. "Elevated Risk Factor', was defined as non-smoker and non-diabetic with total cholesterol 200-240 $\mathrm{mg} \cdot \mathrm{dL}^{-1}$, or untreated systolic blood pressure $140-160 \mathrm{~mm} \mathrm{Hg}$ or untreated diastolic blood pressure 90-100 mm Hg. "Major Risk Factor' 'was defined as current smoker or total cholesterol $\geq 240 \mathrm{mg} \cdot \mathrm{dL}^{-1}$, or treated hypertension, or untreated systolic blood pressure $\geq 160 \mathrm{~mm} \mathrm{Hg}$, or untreated diastolic blood pressure $\geq 100 \mathrm{~mm} \mathrm{Hg}$. burden and lifetime risk for ASCVD, individuals with several traditional risk factors in middle-age do not remain low risk across their remaining lifespan. This can be seen in Figure 1: although all five risk factor groups have relatively similar risk for CVD mortality in the initial 10 years, there are marked differences in risk across the remaining lifespan. We have previously reported that approximately $50 \%$ of all United States adults with low short-term risk are at high risk across their remaining lifespan. ${ }^{12}$

It is within this context that the current study by Hulten et al, in this issue of the Journal of Nuclear Cardiology $^{\circledR}$, should be interpreted. Utilizing data from the Coronary CT Angiography Evaluation for Clinical Outcomes: International Multicenter Registry (CONFIRM), this study observes that high predicted lifetime risk is associated with some, but not all computed tomography derived measurements of coronary artery disease (CAD). ${ }^{13}$ In a cohort of 1,863 asymptomatic individuals in the low Framingham risk category referred for coronary CT angiography, the authors report that high predicted lifetime risk is associated with higher prevalence obstructive and non-obstructive CAD, higher severity scores and higher calcified to partially calcified plaque ratio. In contrast to prior reports, ${ }^{14}$ in this population with a low prevalence of CAC (32\%), high predicted lifetime risk was not associated with higher CAC scores.

The authors also report that high predicted lifetime risk was not associated with differences in short-term coronary heart disease events, including a composite outcome of death, myocardial infarction, or coronary revascularization. With only 11 events, this analysis is underpowered and therefore difficult to interpret. More importantly, it is unclear to what extent lifetime risk stratification can add to short-term risk estimates. Lifetime risk for ASCVD is a function of the same traditional risk factors that influence short-term risk. It is therefore not surprising that lifetime risk stratification did not represent an informative supplement to existing short-term risk estimates. As noted above, the purpose of estimating lifetime risk is not to improve short-term risk prediction but to identify individuals on distinct trajectories and high cardiovascular risk beyond the 10-year window.

Although there is little controversy about the concept of lifetime risk, questions remain regarding its clinical application. For example, what should be done about patients at very low short-term risk (i.e., a low Framingham risk score and zero CAC) but high predicted lifetime risk? The absence of CAC represents a very favorable short-term risk marker, with multiple observational studies confirming virtually the absence of short-term risk in this setting. Although zero CAC is 
informative, we believe that the presence of high predicted lifetime risk cannot be ignored, particularly in light of the robust data from the Cardiovascular Lifetime Risk Pooling Project.

Given that there are no data on the association between CAC and lifetime risk for ASCVD, we sought to answer this very question using data from the Dallas Heart Study. ${ }^{15}$ In this study, we identified 754 participants with serial CAC scans (6.9 years apart) and both low short-term risk and baseline $\mathrm{CAC}=0$. Compared to low lifetime risk, high lifetime risk was associated with nearly a twofold increase in the annualized incidence of CAC. These data suggest that even among individuals with low Framingham risk scores and zero CAC, predicted lifetime risk identifies a subgroup that is more likely to develop subclinical atherosclerosis and in whom long-term risk likely remains elevated. Therefore, high predicted lifetime risk can reliably identify a subgroup of low short-term risk individuals who are on a different risk trajectory, regardless of their current CAC score.

Current guidelines recommend the calculation of lifetime risk for all individuals between the ages of 2039 years and for those between 40 and 59 years without high short-term risk. ${ }^{4}$ These guidelines further suggest that estimates of long-term risk would best be used in the context of a "risk conversation" to guide and motivate non-pharmacological interventions. Thus, lifetime risk estimates can provide a unique opportunity to contextualize the risks related to an unhealthy lifestyle and provide an important perspective, particularly to younger adults. In addition to the role of traditional risk factors described above, our recent work has sought to extend this conversation to include cardiorespiratory fitness, a marker of recent physical activity levels. Using long-term follow-up data from the Cooper Center Longitudinal Study, we were able to create lifetime risk estimates according to age, sex, risk factor burden, and fitness levels in a large number of healthy, middle-aged adults. As shown in Figure 2, midlife fitness levels are associated with robust differences in lifetime risk for cardiovascular mortality, suggesting that fitness levels in middle-age are not just a short-term risk marker but also a marker of lifetime risk ${ }^{16} \mathrm{We}$ believe that more creative use of lifetime risk estimates to target behavior and lifestyle changes would represent an important part of any "risk conversation."

Finally, questions remain regarding the potential to make statin treatment decisions among individuals with low short-term but high lifetime predicted risk. Such an approach is appealing, particularly in light of genetic studies that suggest the dominant role of lifelong reductions in cholesterol in influencing CVD risk. ${ }^{17,18}$ However, there are no long-term data on statin therapy

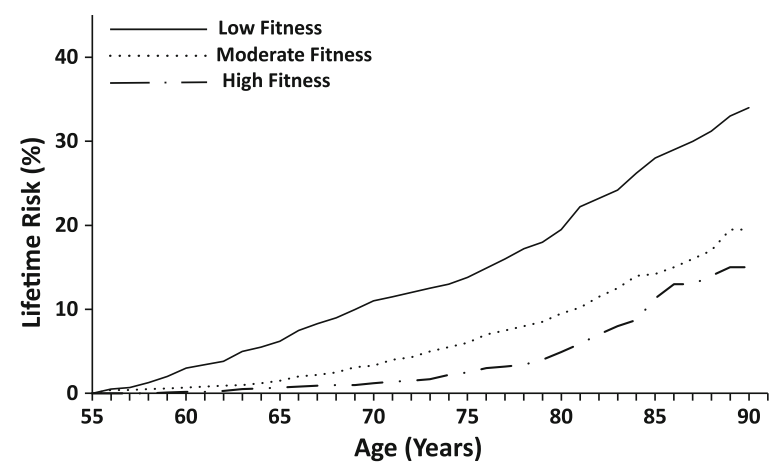

Figure 2. Lifetime risks for cardiovascular disease mortality among men stratified by cardiorespiratory fitness levels measured at age 55 years $(\mathrm{N}=3,424)$. Data derived from the Cooper Center Longitudinal Study, and modified from Berry et $\mathrm{al}^{16}$ Cardiorespiratory fitness categories defined according to Balke treadmill time and categorized according to established age- and sex-specific categories.

and any treatment decision would reflect extrapolation of treatment benefits from short-term clinical trials. Although such a decision would need to be individualized, we believe that primary prevention statin therapy might be reasonable, particularly among middle-aged adults who do not meet current treatment thresholds but who have two or more major risk factors.

In conclusion, the use of short-term risk equations and $\mathrm{CAC}$ both represent useful strategies to estimate short-term risk and also have important implications for short-term treatment decisions. Nevertheless, short-term risk is not complete and is frequently in conflict with the risk across the remaining lifespan. Thus, until we have data on the association between CAC and lifetime risk, we believe that both short-term risk estimates and CAC scores should be interpreted within the context of lifetime risk and, when short-term and lifetime risk estimates are discordant, patients should be counseled on all available strategies to minimize their lifetime risk.

\section{References}

1. 27th Bethesda Conference. Matching the intensity of risk factor management with the hazard for coronary disease events. September 14-15, 1995. J Am Coll Cardiol 1996;27:957-1047.

2. Executive summary of the third report of the National Cholesterol Education Program (NCEP) expert panel on detection, evaluation, and treatment of high blood cholesterol in adults (adult treatment panel III). J Am Med Assoc 2001;285:2486-97.

3. Wood D, De Backer G, Faergeman O, Graham I, Mancia G, Pyorala K. Prevention of coronary heart disease in clinical practice: Recommendations of the Second Joint Task Force of European and other Societies on Coronary Prevention. Atherosclerosis 1998;140:199-270.

4. Goff DC Jr, Lloyd-Jones DM, Bennett G, Coady S, D' Agostino RB $\mathrm{Sr}$, Gibbons R, et al. 2013 ACC/AHA guideline on the assessment of cardiovascular risk: A report of the American College of Cardiology/ 
American Heart Association Task Force on Practice Guidelines. Circulation 2013. doi:10.1161/01.cir.0000437741.48606.98.

5. Stone NJ, Robinson J, Lichtenstein AH, Merz CN, Blum CB, Eckel RH, et al. 2013 ACC/AHA guideline on the treatment of blood cholesterol to reduce atherosclerotic cardiovascular risk in adults: A report of the American College of Cardiology/American Heart Association Task Force on Practice Guidelines. Circulation 2013. doi:10.1161/01.cir.0000437738.63853.7a.

6. Yeboah J, McClelland RL, Polonsky TS, Burke GL, Sibley CT, O'Leary D, et al. Comparison of novel risk markers for improvement in cardiovascular risk assessment in intermediaterisk individuals. J Am Med Assoc 2012;308:788-95.

7. Kavousi M, Elias-Smale S, Rutten JH, Leening MJ, Vliegenthart $\mathrm{R}$, Verwoert GC, et al. Evaluation of newer risk markers for coronary heart disease risk classification: A cohort study. Ann Intern Med 2012;156:438-44.

8. Webber BJ, Seguin PG, Burnett DG, Clark LL, Otto JL. Prevalence of and risk factors for autopsy-determined atherosclerosis among US service members, 2001-2011. J Am Med Assoc 2012;308:2577-83.

9. Enos WF, Holmes RH, Beyer J. Coronary disease among United States soldiers killed in action in Korea: Preliminary report. J Am Med Assoc 1953;152:1090-3.

10. Berenson GS, Srinivasan SR, Bao W, Newman WP, Tracy RE, Wattigney WA, et al. Association between multiple cardiovascular risk factors and atherosclerosis in children and young adults. N Engl J Med 1998;338:1650-6.

11. Berry JD, Dyer A, Cai X, Garside DB, Ning H, Thomas A, et al. Lifetime risks of cardiovascular disease. N Engl J Med 2012;366:321-9.

12. Marma AK, Berry JD, Ning H, Persell SD, Lloyd-Jones DM. Distribution of 10-year and lifetime predicted risks for cardiovascular disease in US adults: Findings from the National Health and Nutrition Examination Survey 2003 to 2006. Circ Cardiovasc Qual Outcomes 2010;3:8-14.

13. Hulten E, Villines TS, Cheezum MK, Berman DS, Dunning A, Achenback S, et al. Calcium score, coronary artery disease extent and severity, and clinical outcomes among low Framingham risk patients with low versus high lifetime risk: Results from the CONFIRM registry. J Nucl Cardiol 2013. doi:10.1007/s12350013-9819-7.

14. Berry JD, Liu K, Folsom AR, Lewis CE, Carr JJ, Polak JF, et al. Prevalence and progression of subclinical atherosclerosis in younger adults with low short-term but high lifetime estimated risk for cardiovascular disease: The coronary artery risk development in young adults study and multi-ethnic study of atherosclerosis. Circulation 2009;119:382-9.

15. Paixao ARAC, Khera A, Berry JD. Cardiovascular lifetime risk predicts incidence of coronary calcification in low risk subjects: Results from the Dallas Heart Study. Circulation 2013;128: A14280.

16. Berry JD, Willis B, Gupta S, Barlow CE, Lakoski SG, Khera A, et al. Lifetime risks for cardiovascular disease mortality by cardiorespiratory fitness levels measured at ages 45,55 , and 65 years in men. The Cooper Center Longitudinal Study. J Am Coll Cardiol 2011;57:1604-10.

17. Ference BA, Yoo W, Alesh I, Mahajan N, Mirowska KK, Mewada A, et al. Effect of long-term exposure to lower low-density lipoprotein cholesterol beginning early in life on the risk of coronary heart disease: A Mendelian randomization analysis. J Am Coll Cardiol 2012;60:2631-9.

18. Cohen JC, Boerwinkle E, Mosley TH Jr, Hobbs HH. Sequence variations in PCSK9, low LDL, and protection against coronary heart disease. N Engl J Med 2006;354:1264-72. 